\title{
Identifying structural and energetic trends in isovalent core-shell nanoalloys as a function of composition and size mismatch
}

\author{
Andrés Aguado a) and José M. López \\ Departamento de Física Teórica, Atómica y Óptica, Universidad de Valladolid, Valladolid 47071, Spain
}

(Received 10 June 2011; accepted 7 September 2011; published online 4 October 2011)

\begin{abstract}
We locate the putative global minimum structures of $\mathrm{Na}_{x} \mathrm{Cs}_{55-x}$ and $\mathrm{Li}_{x} \mathrm{Cs}_{55-x}$ nanoalloys through combined empirical potential and density functional theory calculations, and compare them to the structures of 55-atom Li-Na and Na-K nanoalloys obtained in a recent paper [A. Aguado and J. M. López, J. Chem. Phys. 133, 094302 (2010)]. Alkali nanoalloys are representative of isovalent metallic mixtures with a strong tendency towards core-shell segregation, and span a wide range of size mismatches. By comparing the four systems, we analyse how the size mismatch and composition affect the structures and relative stabilities of these mixtures, and identify useful generic trends. The Na-K system is found to possess a nearly optimal size mismatch for the formation of poly-icosahedral (pIh) structures with little strain. In systems with a larger size mismatch ( $\mathrm{Na}-\mathrm{Cs}$ and $\mathrm{Li}-\mathrm{Cs}$ ), frustration of the pIh packing induces for some compositions a reconstruction of the core, which adopts instead a decahedral packing. When the size mismatch is smaller than optimal ( $\mathrm{Li}-\mathrm{Na})$, frustration leads to a partial amorphization of the structures. The excess energies are negative for all systems except for a few compositions, demonstrating that the four mixtures are reactive. Moreover, we find that $\mathrm{Li}-\mathrm{Cs}$ and $\mathrm{Li}-\mathrm{Na}$ mixtures are more reactive (i.e., they have more negative excess energies) than $\mathrm{Na}-\mathrm{K}$ and $\mathrm{Na}-\mathrm{Cs}$ mixtures, so the stability trends when comparing the different materials are exactly opposite to the trends observed in the bulk limit: the strongly non-reactive Li-alkali bulk mixtures become the most reactive ones at the nanoscale. For each material, we identify the magic composition $x_{m}$ which minimizes the excess energy. $x_{m}$ is found to increase with the size mismatch due to steric crowding effects, and for $\mathrm{Li}_{x} \mathrm{Cs}_{55-x}$ the most stable cluster has almost equiatomic composition. We advance a simple geometric packing rule that suffices to systematize all the observed trends in systems with large size mismatch ( $\mathrm{Na}-\mathrm{K}, \mathrm{Na}-\mathrm{Cs}$, and $\mathrm{Li}-\mathrm{Cs})$. As the size mismatch is reduced, however, electron shell effects become more and more important and contribute significantly to the stability of the Li-Na system. () 2011 American Institute of Physics. [doi:10.1063/1.3645105]
\end{abstract}

\section{INTRODUCTION}

The properties of nanoscale matter cannot usually be inferred from extrapolations of the corresponding bulk properties. Both quantum confinement effects and an increased surface-to-volume ratio often equip nanomaterials with novel structural, catalytic, optical, magnetic, or superatomic properties (just to mention a few examples). Moreover, those properties are strongly size-dependent for nanoparticles with less than a few hundred atoms. Bimetallic nanoparticles (or nanoalloys) provide a promising test ground in this respect because their physical and chemical properties depend not only on cluster size but also on their composition and specific chemical ordering. The additional degrees of freedom result in a rich diversity of structural and electronic behaviors, which can be fine tuned in order to optimize the catalytic selectivity for a particular chemical reaction, for example. Many experimental ${ }^{1-7}$ and theoretical ${ }^{7-40}$ efforts have thus been devoted in recent years in order to understand the properties of nanoalloys at a fundamental level.

The structure, alloying ability, and chemical ordering of bimetallic nanoparticles are not currently well understood

\footnotetext{
a)Electronic mail: aguado@metodos.fam.cie.uva.es.
}

despite the numerous efforts mentioned above. Nanoalloys frequently adopt atomic packing schemes which are different from those observed in the corresponding pure metal clusters. ${ }^{9}$ Also, the bulk phase diagrams do not allow to make predictions about the formation energies of nanoalloys, as many elements which are immiscible in the bulk limit are found to possess negative formation energies below a critical size. ${ }^{39}$ Finally, one would like to know which chemical ordering pattern (core-shell, onion-layered, or randomly mixed) is favored and identify the physical factors responsible for each particular pattern. Immiscible elements in the bulk tend to be separated by a planar interface, but it is often the case that the same two elements form rounded core-shell particles at the nanoscale, adopting thus a segregation pattern which does not minimize the interfacial area. Novel chemical ordering patterns such as the patchy multishell segregation recently observed in Pd-Pt nanoalloys, ${ }^{37}$ or the dynamical coexistence of $\mathrm{Cu}$-rich and $\mathrm{Ag}$-rich facets in $\mathrm{Cu}-\mathrm{Ag}$ nanoalloys, ${ }^{27}$ clearly illustrate the complexity of the structural problem at the nanoscale.

There are several factors that determine the structural preferences of a generic $\mathrm{A}_{x} \mathrm{~B}_{N-x}$ nanoalloy: A large difference in the cohesive energies of the two elements, for example, is a factor opposing mixing and leading to phase 
separation in the bulk limit, where the interfacial energy contribution to stability is negligible. The same factor will favor nanoalloy structures where the number of the strongest homoatomic bonds is maximized; contrary to the bulk, however, the interfacial energy can be comparable to the total energy in a small nanoparticle, and the relative stability of core-shell and Janus-like ${ }^{29,31}$ segregation patterns will depend on the strength of the heteroatomic A-B bonds and also on possible ionic contributions to bonding (charge transfer effects). ${ }^{38}$ A second important factor is the difference in surface energies. The element with lower surface energy will tend to segregate to the surface of the cluster, but depending on the specific composition, this factor alone does not oppose the formation of an alloyed core. ${ }^{20}$ A third, purely geometrical, factor is the size mismatch, which tends to favor structures in which a shell formed by the "big" element surrounds a core formed by the "small" element in order to optimize packing. Reyes-Nava et al. ${ }^{28}$ have recently shown that the repulsive steric effects associated to size mismatch dominate the structural and segregation properties of bimetallic alloys formed by two elements from the same group. The size mismatch effect also helps to relax the stress accumulated in the core region of the most compact atomic packings, favoring the formation of poly-icosahedral or poly-tetrahedral structures. ${ }^{9,11-13}$ Zhang and Fournier ${ }^{17}$ have discussed chemical ordering trends in 55atom bimetallic icosahedral particles as a function of these energetic and geometrical factors. Finally, purely quantum electron shell effects ${ }^{16,36}$ or even magnetic effects ${ }^{15}$ may also have a strong influence on the nanoalloy structures.

In order to gain a more fundamental understanding of the structures and chemical ordering in nanoalloys, it is interesting to analyse a family of similar compounds, identifying if possible structural trends as a function of the several variables mentioned above. In this connection, alkali nanoalloys can be particularly useful as model metallic systems. Both the cohesive and surface energies decrease significantly when moving down the alkali column in the periodic table, so alkali nanoalloys are expected to be representative of isovalent bimetallic particles with a small degree of charge transfer and a strong inclination to surface segregation of the element with bigger size. They also span a very wide range of size mismatches $\Delta a / \bar{a}$ (where $a$ is the bulk lattice constant), from about $19 \%$ in LiNa to about $55 \%$ in LiCs, so they constitute a natural laboratory to study the influence of size mismatch on the structures and stabilities of realistic bimetallic nanoparticles. Doye and Meyer ${ }^{14}$ have already studied the effect of size mismatch on the structures of binary Lennard-Jones clusters, which are considered a model representative of materials where pairwise interactions dominate bonding. Alkali nanoalloys provide a suitable extension of the same idea to systems where many-body metallic interactions prevail.

Very recently, we have reported the putative global minimum (GM) structures of $\mathrm{Li}_{x} \mathrm{Na}_{55-x}$ and $\mathrm{Na}_{x} \mathrm{~K}_{55-x}$ for the full range of compositions. ${ }^{36}$ In this paper, we enlarge our description of alkali nanoalloys by considering mixtures with a much larger size mismatch, namely $\mathrm{Na}_{x} \mathrm{Cs}_{55-x}$ and $\mathrm{Li}_{x} \mathrm{Cs}_{55-x}$. We are mainly interested in identifying trends in the structure and stability of bimetallic nanoalloys as a function of the size mismatch, and for this reason we will offer a systematic compari- son of the four systems, reproducing some of the results from the previous paper when these are needed for a clearer exposition of trends. With increasing size mismatch, we expect that purely geometric packing effects dominate the nanoalloy properties, while electron shell effects may be more important when the size mismatch is small. So another objective of our work is to estimate the relative importance of geometric and electronic shell effects. We focus on 55-atom clusters because the GM structure of the pure alkali clusters is well known, namely a perfect Mackay icosahedron, and it is interesting to analyse the stability of structurally magic clusters upon alloying. ${ }^{8}$ Finally, an added interest of this work comes from the observation that $\mathrm{Li}$ interalkalies exhibit only phaseseparation behavior in the bulk limit, while $\mathrm{Na}-\mathrm{K}$ and $\mathrm{Na}-\mathrm{Cs}$ mixtures form an ordered Laves phase $\left(\mathrm{MgZn}_{2}\right.$ type). Thus the formation energy of Li-alkali bulk mixtures is positive and rapidly increases with the size mismatch, from a value of $56 \mathrm{meV} /$ atom for $\mathrm{Li}-\mathrm{Na}$ to a value of $227 \mathrm{meV} /$ atom for $\mathrm{Li}$ Cs. ${ }^{41}$ Recently, Zhang and Zunger ${ }^{41}$ have shown how the strongly non-reactive Li-Cs mixture becomes reactive and long-range ordered upon the application of an external pressure. In this work, we will show that Li-alkali mixtures also become reactive at the nanoscale, where $\mathrm{Li}-\mathrm{Cs}$ is in fact the most reactive of the four mixtures considered in this paper. The theoretical approach for locating the GM structures is described in Sec. II. Section III describes the structures, stabilities, and also some electronic properties of the nanoalloys and identifies the main trends in these quantities as a function of the size mismatch. Finally, Sec. IV offers some concluding remarks.

\section{COMPUTATIONAL METHODS}

Our computational strategy is exactly the same as in our previous paper on $\mathrm{Li}-\mathrm{Na}$ and $\mathrm{Na}-\mathrm{K}$ mixtures; ${ }^{36}$ so, we provide here just a brief account of it and refer the reader to our previous work for a full exposition of the theoretical method.

In a first stage, we perform unbiased structure optimizations employing the Basin Hopping $(\mathrm{BH})$ method $^{42,43}$ and a Gupta potential ${ }^{44-46}$ description of the potential energy surface. Within this approach, the total energy of an $\mathrm{A}_{x} \mathrm{~B}_{N-x}$ nanoalloy is written as a sum of atomic contributions $E_{i, \alpha}$, where $i=1, \ldots, N$ labels the atom and $\alpha=\mathrm{A}, \mathrm{B}$ the atomic species. Each atomic contribution is in turn decomposed into an attractive band-energy many-body term $E_{i, \alpha}^{\text {band }}$ and a repulsive pairwise contribution $E_{i, \alpha}^{\text {rep }}$

$$
E_{i, \alpha}=E_{i, \alpha}^{\mathrm{band}}+E_{i, \alpha}^{\mathrm{rep}}
$$

$E_{i, \alpha}^{\mathrm{band}}=-\left\{\sum_{j \neq i}^{N} \sum_{\beta=A, B} n_{j \beta} \xi_{\alpha \beta}^{2} \exp \left[-2 q_{\alpha \beta}\left(\frac{r_{i j}}{r_{\alpha \beta}^{0}}-1\right)\right]\right\}^{1 / 2}$

$$
E_{i, \alpha}^{\mathrm{rep}}=\sum_{j \neq i}^{N} \sum_{\beta=A, B} n_{j \beta} A_{\alpha \beta} \exp \left[-p_{\alpha \beta}\left(\frac{r_{i j}}{r_{\alpha \beta}^{0}}-1\right)\right],
$$

where $r_{i j}$ is the distance between atoms $i$ and $j$, and $n_{j \beta}$ is an occupation variable whose value is 1 if atom $j$ belongs to 
species $\beta$ and zero otherwise. Homoatomic interaction (AA and BB) parameters are taken from the work by Li et al. ${ }^{47}$ The heteroatomic parameters are initially expressed as weighted arithmetic averages of the corresponding values for the pure materials, for example,

$$
p_{A B}=\omega p_{A A}+(1-\omega) p_{B B} .
$$

We have tried nine possible $\omega$ values between 0.1 and 0.9 . Test calculations performed for selected compositions showed that the structure of the nanoalloys is quite sensitive to the $r_{A B}^{0}$ value, and that $\omega=0.5$ leads to the best agreement with $a b$ initio results (see below). We thus fix $r_{A B}^{0}=\frac{1}{2}\left(r_{A A}^{0}+r_{B B}^{0}\right)$ in the following. The other four heteroatomic parameters do not seem to have such a strong impact on the predicted structures, because the structures generated by different $\omega$ values are essentially the same (although the relative stabilities of different isomers may of course be dependent on the specific value of $\omega$ ). As the main goal of the empirical potential (EP) calculations in this paper is just to generate a sufficiently diverse set of initial structures as an input for first-principles re-optimization (see below), we have not tried to determine the optimal $\omega$ value. Structural and energetic heteroatomic parameters have been found to be close to the average values for bulk Na-Cs mixtures. ${ }^{48}$ Moreover, the observation that $r_{A B}^{0}$ has a stronger impact on the obtained structures demonstrates that the stable structures are strongly dominated by the huge size mismatch, and this is the reason why the different averages produce essentially the same bank of trial structures once $r_{A B}^{0}$ is fixed. Our conclusion agrees with recent results by Reyes-Nava et al. ${ }^{28}$ who demonstrated that steric effects dominate the structure of bimetallic alloys formed by elements of the same group, simply because the core densities of $\mathrm{A}$ and $\mathrm{B}$ species differ much more than the corresponding valence densities. All the technical details of the $\mathrm{BH}$ runs are the same as in Ref. 36, with only one exception: in this work, we have found it convenient to remove the swap moves (interchanging the identity of two randomly chosen A- and $\mathrm{B}$-atoms) when sampling the potential energy surface. These trial moves always result in isomers with a very high energy, as they oppose the natural tendency of the bigger Cs atoms to segregate to the cluster surface. A similar conclusion has recently been reported by Sicher et al. ${ }^{49}$ in connection with binary Lennard-Jones nanoparticles.

The BH runs provide us with a diverse bank of candidate structures for each value of the composition $x$. Typically, we select 80 different isomers for each composition and re-optimize them at the density functional theory (DFT) level. Following the work of Ferrando et al., ${ }^{18}$ those isomers are selected on the basis of structural descriptors in order to enhance the structural diversity (see Ref. 36 for full details of our procedure). The first-principles calculations themselves have been performed at the Kohn-Sham ${ }^{50}$ DFT (Ref. 51) level. We employ the SIESTA code, ${ }^{52}$ with exchange-correlation effects treated within the spin-polarized local density approximation (we use the Perdew and Zunger parametrization $^{53}$ of the electron gas results of Ceperley and Alder ${ }^{54}$ ), and norm-conserving pseudopotentials to describe the core electrons. ${ }^{55,56}$ The basis set is of DZP (double zeta plus polarization) quality. Other technical parameters of the SIESTA runs are the same as in Ref. 36.

Nanoalloy stability is analysed here in terms of the excess energy (which sometimes is alternatively called mixing energy): ${ }^{7,8,15}$

$E_{\mathrm{exc}}\left(A_{x} B_{55-x}\right)=E\left(A_{x} B_{55-x}\right)-x \frac{E\left(A_{55}\right)}{55}-(55-x) \frac{E\left(B_{55}\right)}{55}$,

where $E\left(A_{55}\right)$ and $E\left(B_{55}\right)$ are the energies of the pure 55-atom clusters in their GM structure. The excess energy is zero for the pure clusters by definition. Negative excess energy values indicate that formation of the corresponding nanoalloy is energetically favorable. Although other stability measures are available for nanoalloys, ${ }^{40}$ and some of them were calculated in our previous work, ${ }^{36}$ the excess energy will suffice to describe the system-dependent trends which are the main goal of this paper.

As a measure of cluster size, we employ the root-meansquare radius $r$ obtained from

$$
r^{2}=\frac{1}{N} \sum_{i}^{N}\left(\left(x_{i}-x_{C}\right)^{2}+\left(y_{i}-y_{C}\right)^{2}+\left(z_{i}-z_{C}\right)^{2}\right),
$$

which quantifies the average cluster radius with respect to the centroid $\left(x_{C}, y_{C}, z_{C}\right)$ of nuclear positions. An excess radius is then defined as

$$
r_{\mathrm{exc}}\left(A_{x} B_{55-x}\right)=r\left(A_{x} B_{55-x}\right)-x \frac{r\left(A_{55}\right)}{55}-(55-x) \frac{r\left(B_{55}\right)}{55} \text {. }
$$

Obviously, the excess radius is also zero for the pure clusters. A negative excess radius implies that the nanoalloy has a more compact structure as compared to an ideal mixture. We will see in Sec. III that this parameter is very useful for rationalizing the structural and stability trends in alkali nanoalloys. The cluster shape is quantified by using the Hill-Wheeler parameters, ${ }^{57}$ as in our previous work. ${ }^{36}$

Vertical ionization potentials (VIPs) have been calculated as a measure of the electronic stability of the putative GM structures. Partial atomic charges have also been evaluated in order to analyse possible charge transfer contributions ${ }^{38}$ to nanoalloy stability. We have chosen two methods which are based on a direct partitioning of the electron density distribution (and are thus quite insensitive to basis set issues): the Voronoi deformation density (VDD) charges ${ }^{58}$ and the atomsin-molecules Bader charges, ${ }^{59}$ as modified by Spackman and Maslen. ${ }^{60}$ Both methods obtain atomic charges by integrating the so-called deformation density (the difference between the molecular and promolecular densities ${ }^{58}$ ) over a certain atomic region, and differ only in the definition of the atomic boundaries (see Ref. 36 for more details of these calculations).

\section{RESULTS}

We offer in first place a brief summary of the structures obtained for $\mathrm{Li}_{x} \mathrm{Na}_{55-x}$ and $\mathrm{Na}_{x} \mathrm{~K}_{55-x}$ nanoalloys. ${ }^{36}$ This will be useful when comparing the different alkali mixtures. For those systems, we essentially observed a competition between two structural motifs: at dilute concentrations of 
one of the atomic species, the structure of the 55-atom pure metal clusters (a perfect Mackay icosahedron) is preserved, i.e., the atoms of the minority component are incorporated into the icosahedron (Ih) as substitutional impurities. At intermediate compositions, a poly-icosahedral (pIh) packing is adopted, and the structures show core-shell segregation, with the atomic species of smaller size preferentially occupying the cluster core (core and surface atoms are distinguished on the basis of an analysis of coordination numbers and also of the distribution of atomic distances to the cluster center $\left.{ }^{61}\right)$. The pIh structures show strong prolate deformations, as predicted by the jellium model for metal clusters with 55 electrons. ${ }^{62}$ The electron shell contribution seems thus to be a factor determining the shape of the pIh structures. The compositions for which the Mackay icosahedron is stable are $x=1-9,45-54$ for Li-Na and $x=1-5,46-54$ for Na-K. The stability range of the pure cluster structure is thus wider for the system with a smaller size mismatch. The pIh structures of $\mathrm{Na}_{x} \mathrm{~K}_{55-x}$ have a high structural order, as measured by the point group symmetries and a small dispersion in the bond lengths, while those of $\mathrm{Li}_{x} \mathrm{Na}_{55-x}$ undergo a partial amorphization away from the perfect $\mathrm{p}$-Ih shapes.

\section{A. Structures of $\mathrm{Na}_{x} \mathbf{C s}_{55-x}$}

Figure 1 shows a selection of the putative GM structures of $\mathrm{Na}_{x} \mathrm{Cs}_{55-x}$. For Cs-rich clusters, the Mackay icosahedron is stable only up to $x=3$. This range of compositions is clearly dominated by the more abundant Cs-Cs bonds and by the high structural stability of the host icosahedron. The large size mismatch does not favor substitutional doping, however, as this results in too long $\mathrm{Na}-\mathrm{Na}$ distances and generates a substantial strain in the host structure. In the composition range $x=4-11$, the structures have no rotational symmetries. The number of core atoms gradually increases from 14 for $x=4$ to 16 for $x=11$. The core itself shows a clear segregation of the two atomic species, with the Na subsystem occupying an off-center position within the structure. This induces an interesting Janus-like growth pattern, with the Cs

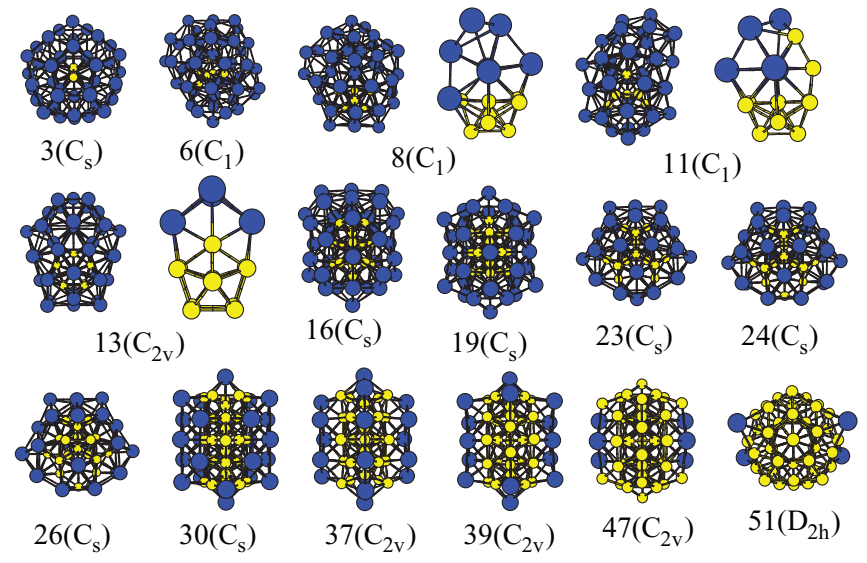

FIG. 1. A representative selection of the putative GM structures of $\mathrm{Na}_{x} \mathrm{Cs}_{55-x}$ nanoalloys, as predicted by the DFT calculations. The composition $x$ and the point group symmetry are given below each isomer. For $x=8,11$, and 13 , we separately show the cluster core. Sodium and cesium atoms are represented by yellow and blue colors, respectively. shell forming an anti-Mackay (AM) overlayer on the Na-rich side but a Mackay overlayer on the Cs-rich side of the core. Although the Na-Na bond lengths are more reasonable, the embedded $\mathrm{Na}$ cluster is not yet very compact (the number of $\mathrm{Na}-\mathrm{Na}$ bonds is not maximized-see the structure for $x=11$ as an example-), demonstrating that $\mathrm{Na}-\mathrm{Cs}$ and $\mathrm{Cs}-\mathrm{Cs}$ bonds still dominate the stability. In fact, for $x=12-13$ we observe a reconstruction of the core, which has 17 atoms and adopts a decahedral packing in order to provide a favorable seed for the growth of the Cs shell. The GM structures of pure sodium clusters with 12-13 atoms are completely different, ${ }^{63}$ and based on pIh packing. The less dense decahedral packing allows to form a compact high-symmetry shell with not too strained Cs-Cs distances. We notice that decahedral cores were not observed in $\mathrm{Li}-\mathrm{Na}$ and $\mathrm{Na}-\mathrm{K}$ mixtures, ${ }^{36}$ so the core reconstruction seems to be favored only for sufficiently large size mismatches.

For $x=14-28$, the strongest Na-Na bonds already dominate the energetics and the core always adopts a compact pIh structure, which is covered by an AM overlayer formed by Cs atoms. The structures generally have a high structural order and for many compositions a mirror-plane symmetry, like those obtained for $\mathrm{Na}_{x} \mathrm{~K}_{55-x} \cdot{ }^{36}$ There is, however, a significant difference: many of the Na-Cs structures in this range, mostly those around the magic composition located at $x=24$ (see Sec. III C), have a strong oblate deformation, which is in contrast with the prolate shapes observed for $\mathrm{Li}-\mathrm{Na}$ and Na-K and favored by the electron shell structure. ${ }^{62}$ This observation suggests that electron shell closing effects become of secondary importance with increasing size mismatch, and that purely geometric packing arguments dictate the shape of the most stable structures. The stability range of the prolate pIh structures is reduced to compositions $x=29-50$ for $\mathrm{Na}_{x} \mathrm{Cs}_{55-x}$ nanoalloys, a narrower range as compared to Li-Na and Na-K mixtures. ${ }^{36}$ These structures are, therefore, penalized by a large size mismatch. They are based on an elongated $\mathrm{pIh}_{31}$ core, formed by interpenetrating four $\mathrm{Ih}_{13}$ units along a single direction (the vertical direction in Figure 1). The $\mathrm{Cs}$ atoms cannot anymore provide a complete overlayer, so the number of $\mathrm{Na}$ atoms exposed to the cluster surface increases rapidly with $x$. Finally, for $x=51-54$, the Mackay icosahedron is recovered, with the substitutional Cs impurities located at the vertex sites of the outermost shell.

\section{B. Structures of $\mathrm{Li}_{x} \mathbf{C s}_{55-x}$}

Figure 2 shows a sample of the structures adopted by $\mathrm{Li}_{x} \mathrm{Cs}_{55-x}$ nanoalloys. The stability of the perfect Mackay icosahedron is further reduced to only two compositions, namely, $x=1$ and $x=54$ (not explicitly shown). The size mismatch is now so large that for $x=2-3 \mathrm{a} \mathrm{Li}_{2}$ dimer substitutes a single Cs atom. A series of quite amorphous structures, with the embedded lithium cluster in an off-center position, is observed for $x=4-14$, a slightly wider range as compared to the Na-Cs system. An exception occurs only for $x=11$, which shows a higher structural order and an approximate 6-fold rotational symmetry axis (the point group is nevertheless $\mathrm{C}_{1}$ because one of the lithium atoms in the core breaks 

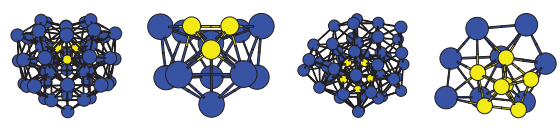

$3\left(\mathrm{C}_{\mathrm{s}}\right)$
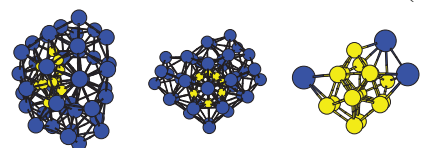

$7\left(\mathrm{C}_{1}\right)$

$16\left(\mathrm{C}_{1}\right)$

$$
12\left(\mathrm{C}_{1}\right)
$$

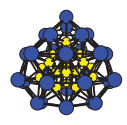

$25\left(\mathrm{C}_{\mathrm{s}}\right)$

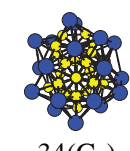

$34\left(\mathrm{C}_{1}\right)$

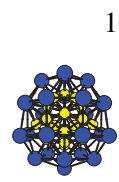

$29\left(C_{s}\right)$
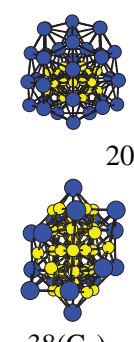

$38\left(\mathrm{C}_{2}\right)$

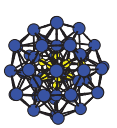

$11\left(\mathrm{C}_{1}\right)$
FIG. 2. A representative selection of the putative GM structures of $\mathrm{Li}_{x} \mathrm{Cs}_{55-x}$ nanoalloys, as predicted by DFT calculations. The point group symmetry and the composition $x$ are given below each isomer. For some compositions, the cluster core is shown separately. Lithium and cesium atoms are represented by yellow and blue colors, respectively.

the perfect symmetry). The number of core atoms gradually increases from 14 for $x=4$ to 18 for $x=14$.

The structures with a decahedral core are found to be much more stable for Li-Cs as compared to Na-Cs, and dominate the composition range $x=14-25$. An increasing size mismatch thus favors the formation of less densely packed core structures in core-shell nanoalloys. For some compositions, we indeed observe poly-decahedral packing, obtained by interpenetrating several 13-atom decahedra. For $x=20$, the lithium subsystem is formed by two interpenetrated $\mathrm{Li}_{13}$ decahedra, while three decahedra form the core of the highsymmetry structure observed for $x=25$. Close to the stability limit of the decahedral family, we observe a close competition of poly-decahedral and pIh core motifs. For example, $x=23$ is an exception within the range $x=14-25$ and has a pIh core because 23 is a structurally magic number for pIh growth. $x=23$ is also the first composition for which a core-shell segregated structure (without $\mathrm{Cs}$ atoms in the core) is formed. All the structures in the composition range $x=26-37$ contain a pIh core and have quite spherical shapes. The magic composition is located at $x=29$ (see Sec. III C) and this cluster is very close to perfectly spherical, demonstrating again that atomic packing effects dominate over electronic shell effects. The structures in this range show a frustrated pIh packing: the Cs shell cannot form a perfect AM overlayer due to the huge size mismatch, and it consists of a mixture of 5-fold and 4-fold rings of Cs atoms, as seen most clearly in Figure 2 for $x=29$. The frustration is reflecting the difficulty of efficiently packing the much bigger Cs atoms around the very small core of $\mathrm{Li}$ atoms, and it is indeed surprising that the $x=29$ structure is so highly structured and symmetric. This is a nice example demonstrating that the high structural freedom in nanoscale materials can result in emergent structural order rules, even in cases of strong geometric frustration. Structures containing an elongated pIh shape are now further restricted to compositions $x=38-52$, and moreover the degree of prolate deformation is the smallest of the four bialkali systems (see for example the structure for $x=42$ in Figure 2, where the Cs atoms cap the elongated structure lat- erally, resulting in a smaller prolate deformation). Finally, for $x=53$ the core of the structure is based on the Z14 FrankKasper polyhedron ${ }^{43}$ and contains two disclination lines. It is interesting to mention that this kind of structure is predicted to be stable for a wider range of compositions and for the four materials studied according to the Gupta potential, ${ }^{36}$ but it is strongly penalized at the DFT level, where it only survives for a single system ( $\mathrm{Li}-\mathrm{Cs}$ ) and, moreover, for a single composition.

The atomic coordinates of the putative GM structures located in this work are reported in the supplementary material. ${ }^{64}$ There we include not only the DFT structures but also the Gupta structures, which have not been explicitly discussed here. Very briefly, we notice that the accuracy of the Gupta potential for structure prediction improves with an increasing size mismatch, which is already an indication that electron shell effects (not accounted for by an empirical potential) are less important for these systems. For example, all the Gupta local minima remained stable after DFT reoptimization, and there is only a small discrepancy between the Gupta and DFT predictions for the average A-A, B-B, and A-B distances. The energetic ordering of isomers is almost always different in Gupta and DFT calculations, although we could not identify any systematic trend in the deviations (the EP may predict a decahedral core when DFT predicts an icosahedral core or vice versa). In any case, many of the DFT GM structures of $\mathrm{Li}_{x} \mathrm{Cs}_{55-x}$ are found within the 10 most stable isomers predicted by the Gupta model, mostly for compositions around the magic one. In particular, this observation confirms that a finer tuning of the heteroatomic Gupta parameters is not needed for the main purposes of this work.

We have only described the GM structures up to this point. However, we have generated a large number of structural isomers for each material and composition, whose properties are briefly discussed now. In particular, the energy difference between the two most stable structures, $E_{12}$, is sometimes quoted as an indication of the thermal stability of a cluster. ${ }^{40}$ Although we agree that $E_{12}$ may be a useful quantity, the information it provides about thermal stability is necessarily incomplete, as it is the whole spectrum of isomer energies which ultimately controls the thermal behavior. For example, it is often found that the structure of the first excited isomer is very similar to the GM structure, while more different structural motifs have substantially higher energies. In such a situation, the presence of one (or a few) isomers very similar to the GM structure will just account for the floppy character of the GM geometry at low temperatures, but that structural motif (floppy in character) may be stable up to significantly higher temperatures (see a recent simulation of the melting of $\mathrm{Na}_{41}^{+}$as a specific example ${ }^{65}$ ), so that $E_{12}$ alone will not correlate in general with the melting temperature or other thermal properties.

Concerning the alkali nanoalloys studied in this work, we have generally found isomers with energies just $0.02 \mathrm{eV}$ or less above the GM energy. These isomers are, with few exceptions, very similar to the GM structure. For example, for $x>x_{m}$, where both big and small atoms occupy the outermost shell, the low-energy excitations are typically homotops, where the A and B species occupy slightly different sites 

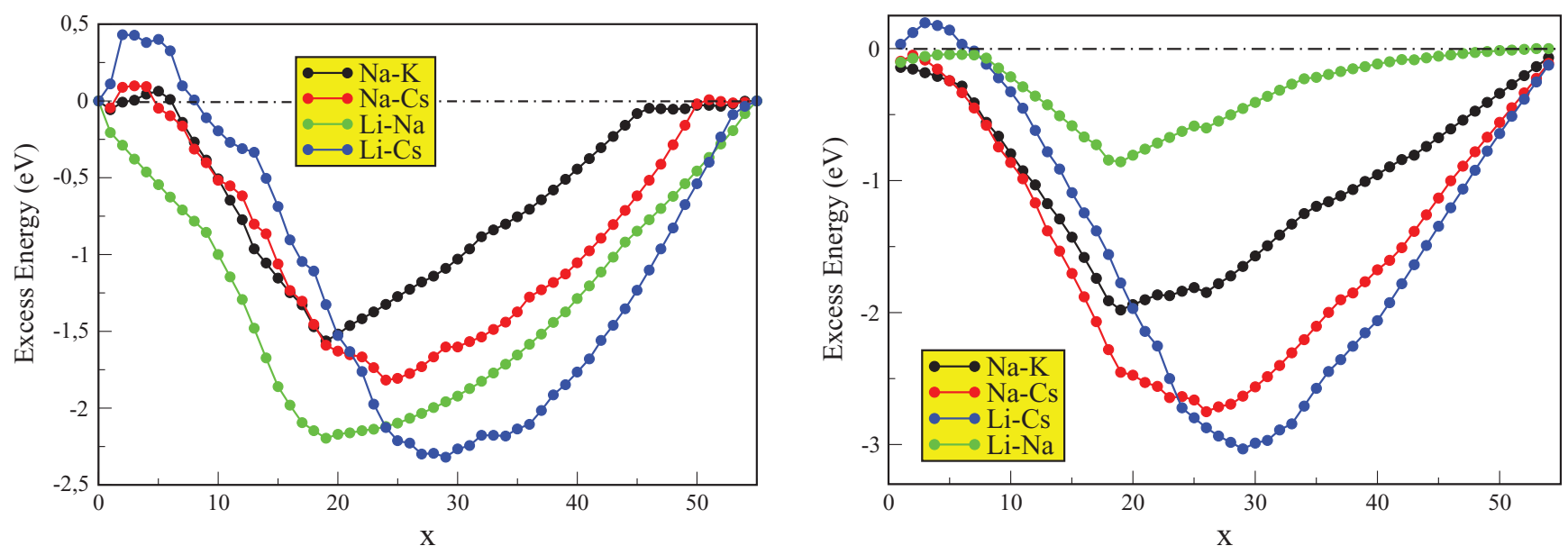

FIG. 3. The excess energies of the four alkali nanoalloys are shown as a function of composition for the DFT (left plot) and Gupta (right plot) levels of theory. The results for Li-Na and Na-K mixtures are taken from Ref. 36.

within the shell. We speculate that these homotops will coexist in a statistical sense at low temperatures but most probably not in a dynamical sense (on the time scale of typical cluster beam experiments) because of large energy barriers. For very small $x$ values, the low-energy excitations usually imply the redistribution of one or a few atoms of the shell (formed in this case only by the big atoms). We speculate that this kind of isomer energy spectrum may lead to surface disorder or even surface melting at relatively low temperatures. On the other hand, isomers with a clearly different structure (for example, those having a different core motif or a very different cluster shape) are usually found only at energies $0.07 \mathrm{eV}$ or more above the GM energy.

\section{Stability trends in binary alkali nanoalloys}

Figure 3 shows the excess energies of the GM structures, both at the DFT and Gupta levels of theory. The excess energies are negative except at some dilute concentrations of the atomic element with smaller size, so the formation of the nanoalloys is generally an exothermic process. The magical compositions are $x_{m}=19,19,24$, and 29 for Li-Na, Na-K, $\mathrm{Na}-\mathrm{Cs}$, and Li-Cs mixtures, respectively. With the only exception of Li-Na mixtures, $x_{m}$ is thus seen to increase with the size mismatch. The Gupta potential results reproduce this qualitative trend and even predict the correct $x_{m}$ values, except for Na-Cs where the Gupta model prediction $\left(x_{m}=26\right)$ is shifted by two units as compared to the DFT results.

Let us focus now on the magic compositions and compare the different materials. Li-Cs is found to be the most reactive mixture, followed by $\mathrm{Li}-\mathrm{Na}, \mathrm{Na}-\mathrm{Cs}$, and $\mathrm{Na}-\mathrm{K}$. Surprisingly, this stability ordering is exactly the opposite to the one observed in bulk solid mixtures ${ }^{41,66}$ where moreover the Li-alkali mixtures are strongly non-reactive and, thus, have positive formation energies. The relative stabilities of the different mixtures are correctly predicted by the Gupta potential with the only exception of the Li-Na mixtures, which would have the lowest stability according to the EP calculations. The comparison between Gupta and DFT results strongly hints at the possibility of a significant electron shell contribution to the stability of Li-Na nanoalloys, while in the other alkali mixtures the stability would be dominated by structural effects, which are reasonably accounted for by the EP model.

The excess radii are shown as a function of composition in Fig. 4. We show only the DFT results because the Gupta predictions are very similar. The nanoalloys have negative excess radii for most compositions, i.e., they are more compact than an ideal mixture of the pure metal clusters. For each separate material, the most stable and the most compact compositions coincide. This demonstrates that the main source of stabilization is structural, and results from an optimization of the atomic packing. The minimum excess radius is thus pointing at that composition for which the core and the shell are most tightly bound. Indirectly, this is suggesting that the interfacial energy provides an essential contribution to the stabilization of the core-shell chemical ordering, which does not minimize the interfacial area, even if the two species are segregated. The Li-Na mixture is once more peculiar, because the nanoalloy with composition $x=45$ is only marginally less compact

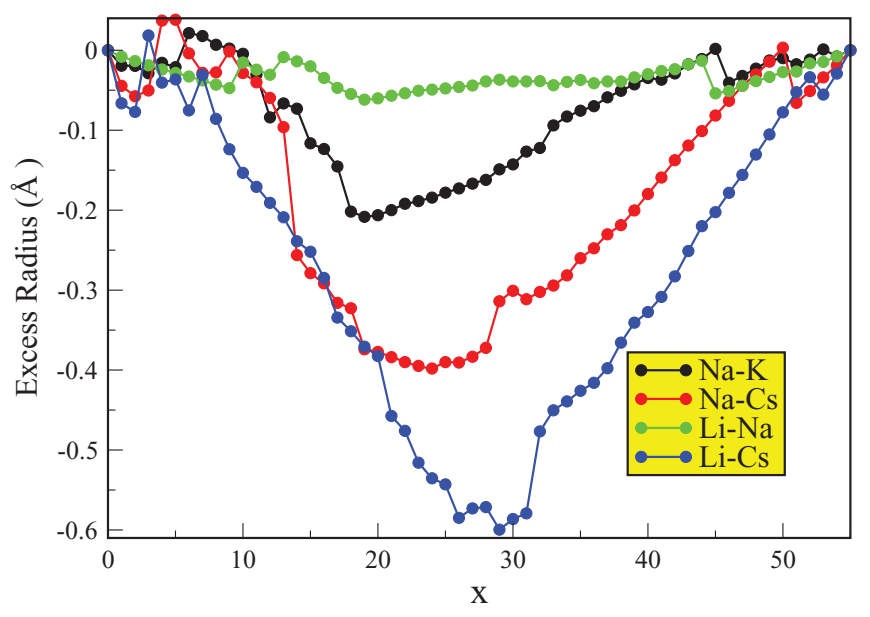

FIG. 4. Excess radii of four different alkali nanoalloys as a function of composition, calculated at the DFT level. The results for $\mathrm{Li}-\mathrm{Na}$ and $\mathrm{Na}-\mathrm{K}$ mixtures are taken from Ref. 36. 
than the magic composition $x_{m}=19$ and is nevertheless much less stable. Therefore, there is a lower correlation between structural compactness and stability for this material.

Focusing now on the magic compositions, we find that a material is more stable the higher its compactness degree, with the only exception of the Li-Na system. Therefore, Li-Cs is the most reactive system at the nanoscale simply because it is the most compact one as compared to an ideal solution. The excess radius is thus found to be a very powerful parameter, which is able to rationalize both the composition dependence of the excess energies for each separate material and the relative stabilities of the different systems for sufficiently large size mismatches. The Li-Na system shows the lowest compactness degree, which does not correlate with its DFT stability but correlates with the low stability predicted at the Gupta level of theory. The Gupta model thus predicts correct structural trends but incorrect energetic trends for the Li-Na system. This is already a quite clear demonstration that electron shell effects must be the source of the additional stabilization observed in Li-Na at the DFT level.

The analysis of atomic charges reveals a very small degree of charge transfer in Li-Na and Na-K. ${ }^{36}$ Li-Cs has the largest electropositivity difference, but even for this system the charge transfer is quite small. For example, at the magical composition $x_{m}=29$, the average charges on Li and Cs atoms are, respectively, -0.07 and +0.06 . Both the Voronoi and Bader methods result in similar charges, so we believe the predicted charge transfer picture is reliable. Although small, the charge transfer strengthens the bonding between core and shell, contributing to the higher stability of Li-Cs nanoalloys. The magnitude of the charge transfer increases at the dilute limits: for example, in $\mathrm{Li}_{1} \mathrm{Cs}_{54}$, the charge on the innermost $\mathrm{Li}$ atom is -0.2 , larger than the average Li charge in $\mathrm{Li}_{29} \mathrm{Cs}_{26}$. This charge comes from the first coordination shell around the lithium atom, which is formed by 12 Cs-atoms with a charge of +0.04 each. These atoms, thus, also tranfer 0.28 electrons to the Cs atoms in the outermost surface shell, which become negatively charged. In summary, minority atoms become more highly charged at the dilute limits, while the charge on the majority atoms becomes both positive and negative. This kind of oscillating response observed in the electron density is typical for a metal around a defect. Finally, we notice that the charge transfer in pressurized bulk Li-Cs alloys is much more important, as it may be as big as 0.5 electrons at $160 \mathrm{GPa}$, forming an "ionic metal" 41 with long-range order. The physical factors explaining the reactivity of Li-Cs nanoscale mixtures are therefore very different from those operating in the pressurized bulk mixtures.

In order to illustrate the importance of electron shell effects for the stability of Li-Na nanoalloys, we show in Figure 5 the VIP as a function of composition for the different nanoalloys. The excess VIP is found to be large and negative for all systems except for Li-Na. A negative excess VIP indicates that the highest occupied molecular orbital (HOMO) is destabilized by the nanoalloy formation process. In fact, for both Na-Cs and Li-Cs, the HOMO at the magic composition has essentially the same energy as the HOMO of the pure $\mathrm{Cs}_{55}$ cluster. This is because the most external part of the HOMO is completely dominated by the Cs atoms which form the shell of the nanoalloy. For Li-Na, however, the excess VIP is positive for many compositions including the magic one, suggesting that both $\mathrm{Na}$ and $\mathrm{Li}$ atoms contribute to the tail region of the HOMO. Li-Na nanoalloys are, therefore, the only systems showing a significant electronic stabilization. Nevertheless, the magic composition $\left(x_{m}=19\right)$ does not belong to the composition interval $x_{m}=30-44$, where the largest VIP values appear; so, the magic composition for $\mathrm{Li}-\mathrm{Na}$ results from a competition between structural and electronic effects.

In the supplementary material, ${ }^{64}$ we provide a detailed analysis of average interatomic distances for a few selected

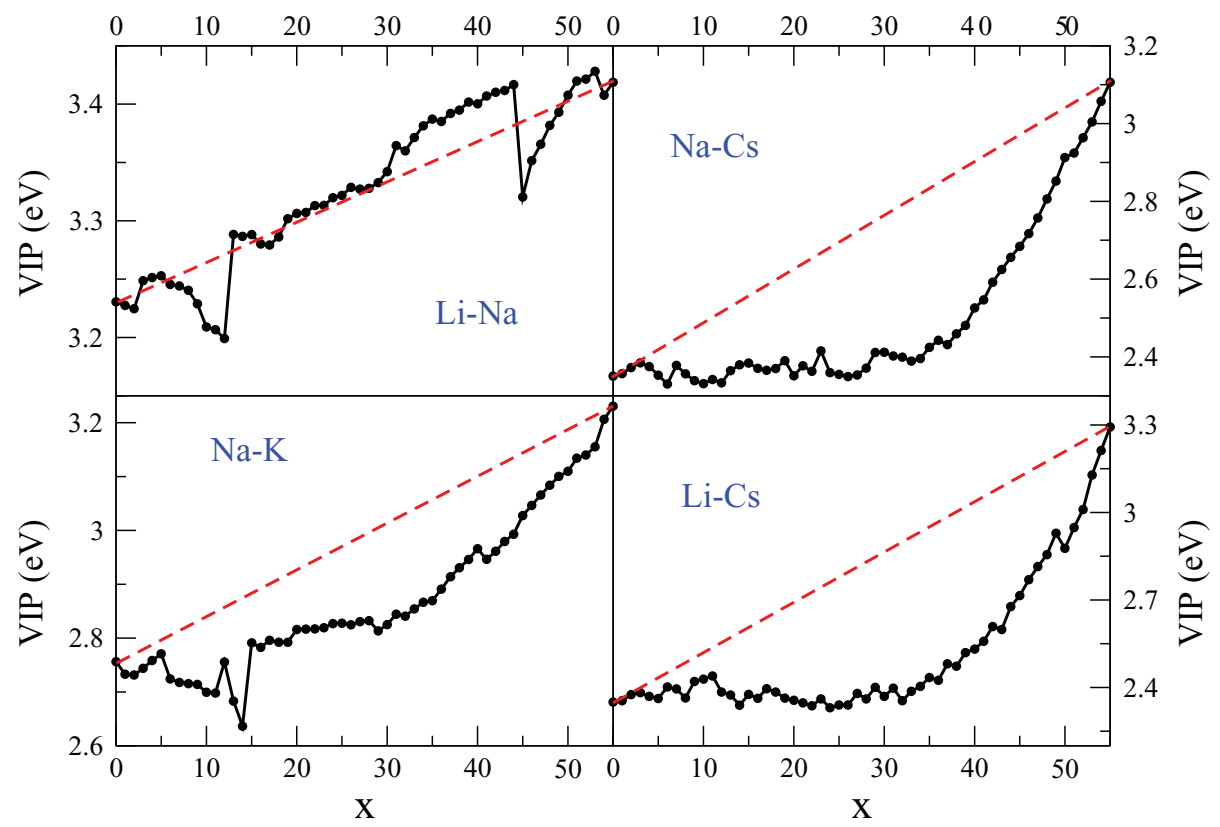

FIG. 5. The vertical ionization potential of four different alkali nanoalloys is plotted as a function of composition. The results for the pure clusters $(x=0$ and 55) are also included. The dashed red line is a linear interpolation between the two pure clusters. The difference between the VIP and that line is the excess VIP. The results for Li-Na and Na-K mixtures are taken from Ref. 36. 

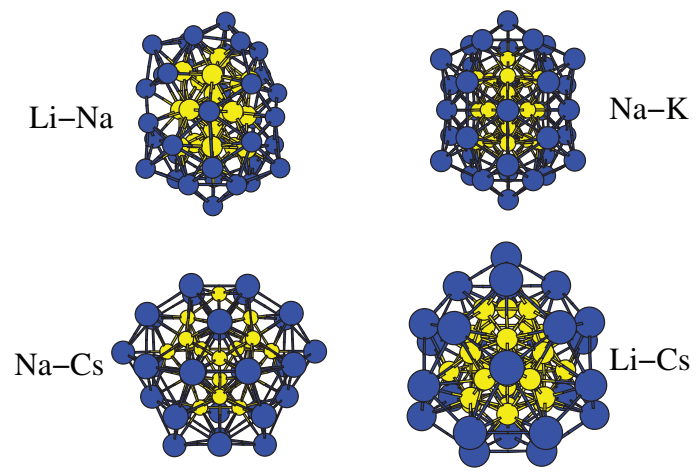

FIG. 6. A summary plot showing the putative GM structures for the magic compositions of the four alkali nanoalloys.

compositions. The analysis confirms that the most stable composition $x_{m}$ achieves, for each material, not only optimal heteroatomic distances (as demonstrated above by the excess radius curves) but also optimal homoatomic distances. The same analysis is also helpful in rationalizing the structural differences between Li-Na and Na-K. Although both nanoalloys have the same magic composition and a similar structure, Figure 6 clearly shows that the structure of $\mathrm{Na}_{19} \mathrm{~K}_{36}$ is highly ordered, while that of $\mathrm{Li}_{19} \mathrm{Na}_{36}$ undergoes a partial amorphization which increases the number of vacancies in the sodium shell, so more internal lithium atoms are exposed to the surface. The results in Table S-II show that both $\mathrm{Na}-\mathrm{Na}$ and $\mathrm{K}-\mathrm{K}$ bond lengths have close to optimal values in $\mathrm{Na}_{19} \mathrm{~K}_{36}$. The Na-K system thus has a size mismatch which is suitable to build perfect pIh structures with little strain. On the contrary, Na-Na bonds are too stretched when we constrain $\mathrm{Li}_{19} \mathrm{Na}_{36}$ to adopt an ordered pIh structure (see Table S-II). The tension in the Na-Na bonds puts the lithium core under pressure, and as a result the $\mathrm{Li}-\mathrm{Li}$ distances are too compressed. It is the tendency of Na-Na bonds to shrink which induces a partial amorphization because a perfect pIh epitaxy is no longer possible. The amorphization relaxes the core stress without modifying the optimal core-shell distance and, so, results in a more stable structure. We notice that neither sodium nor lithium pure clusters have a strong tendency to form amorphous structures, ${ }^{67}$ so the amorphization of Li-Na nanoalloys is a synergistic effect, which results from a size mismatch which is slightly smaller than needed for a perfect pIh packing.

Figure 7 shows the partial electronic densities of states (PDOS) for both ordered and disordered isomers of $\mathrm{Na}_{19} \mathrm{~K}_{36}$ and $\mathrm{Li}_{19} \mathrm{Na}_{36}$. The DOS has been obtained by broadening the KS eigenvalues with gaussian functions of width $0.06 \mathrm{eV}$. Each PDOS curve has been normalized to unity, so it represents the average contribution of a single atom to the
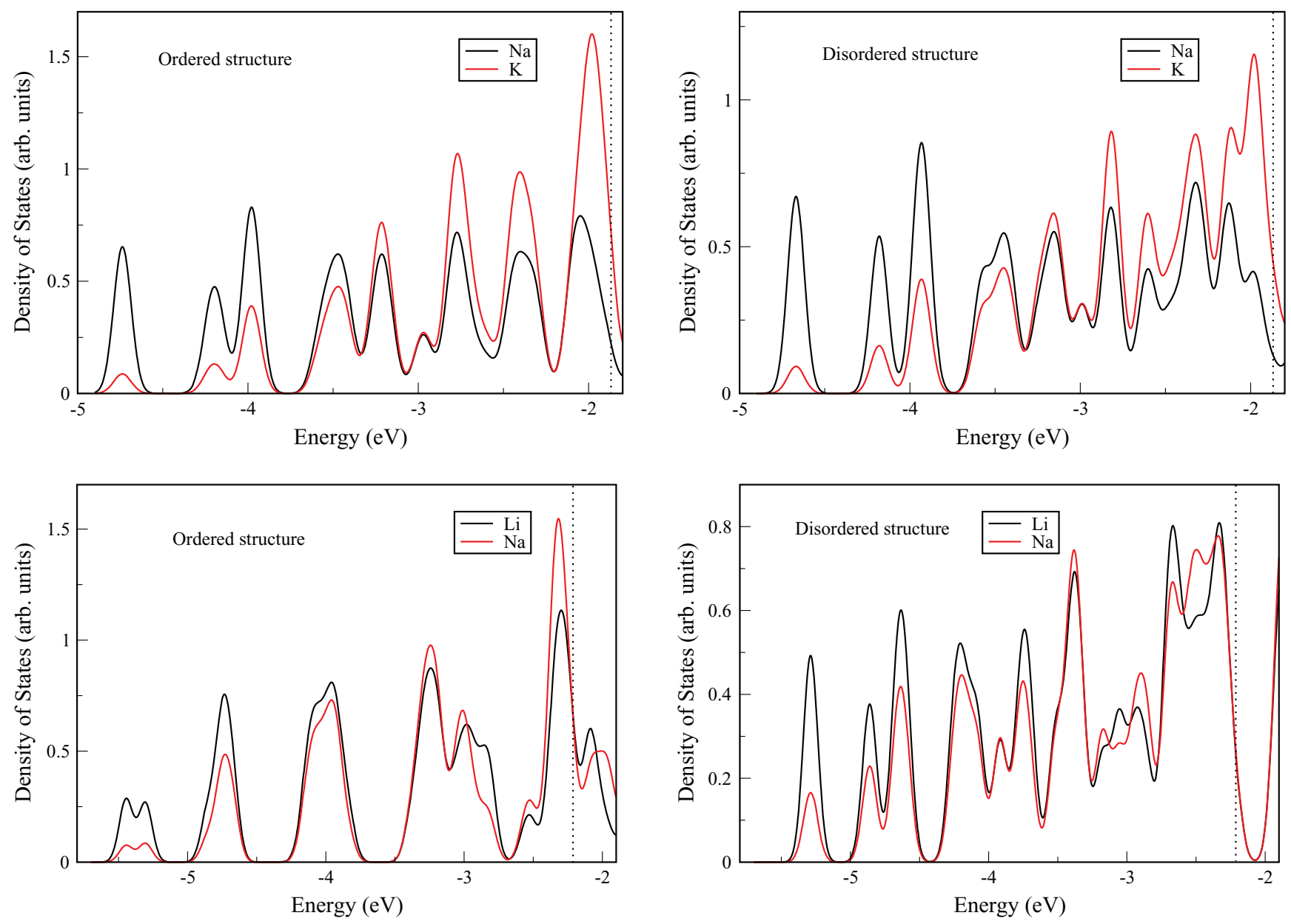

FIG. 7. The partial DOS of each atomic component are shown for both the ordered and amorphous isomers of $\mathrm{Na}_{19} \mathrm{~K}_{36}$ and $\mathrm{Li}_{19} \mathrm{Na}_{36}$. All curves have been normalized, so they represent the average contribution of a single atom to the total DOS. The dotted line marks the position of the HOMO. 
total DOS. The PDOS of $\mathrm{Na}_{19} \mathrm{~K}_{36}$ have similar features for both isomers: sodium atoms contribute more to the innermost "core" bands, while $\mathrm{K}$ atoms clearly dominate the outermost "valence" bands. This is the reason why the VIP of $\mathrm{Na}_{19} \mathrm{~K}_{36}$ is very similar to the VIP of $\mathrm{K}_{55}$. Moreover, both isomers have an open electronic shell because the electron shell closing and the concomitant gap in the DOS occur at 58 electrons, as predicted by the jellium model. The KS eigenvalues are quite spread in energy which results in broad and overlapping bands in the DOS for both isomers. Na-Cs and Li-Cs systems are not shown explicitly because the PDOS curves are very similar to the results for $\mathrm{Na}-\mathrm{K}$.

The situation is quite different, however, for the Li-Na mixture. Focusing on the ordered isomer, it is first appreciated that the bands in the DOS are narrower as compared to the other systems, pointing to an unfavorable electronic kinetic energy contribution; in second place, the $\mathrm{Na}$ atoms do not dominate the outermost bands so strongly as in the other mixtures, because in $\mathrm{Li}-\mathrm{Na}$ the core-shell distance is not large compared to the spatial extension of the lithium orbitals; finally, although the main electron shell closing continues to occur for 58 electrons, a pseudogap opens at 56 electrons, that is, just after the HOMO energy, and the LUMO has a predominant contribution from $\mathrm{Li}$ atoms. The amorphization shifts the LUMO to higher energies and so shifts the electron shell closing from 58 to 56 electrons, opening a gap of $0.4 \mathrm{eV}$ in the DOS. $\mathrm{Li}_{19} \mathrm{Na}_{36}$ is thus almost a closed-shell system, and, so, very stable from the electronic point of view. $\mathrm{Na}$ and $\mathrm{Li}$ atoms contribute now evenly to the outermost DOS bands, explaining the trends in the excess VIP. This is due to the generation of more vacancies in the shell which expose more $\mathrm{Li}$ atoms to the surface. The shift of the LUMO to higher energies is also related to the additional vacancies in the shell: a detailed analysis of the LUMO shows that it receives the largest contributions from those $\mathrm{Na}$ and $\mathrm{Li}$ atoms which are close to a vacancy. The electron density of the LUMO is, therefore, partially localized at the vacancies, which lack a nuclear attractor and are therefore a less stable environment for the electrons. Finally, the amorphization induces a significant broadening of the DOS bands, which show a higher degree of overlap in the disordered structure.

The amorphous structure is $0.5 \mathrm{eV}$ more stable than the ordered one in Li-Na, while it is destabilized by $0.4 \mathrm{eV}$ in $\mathrm{Na}-\mathrm{K}$. These are quite substantial energy differences. For both systems, the amorphization lowers the electronic kinetic energy but raises the potential energy. In Na-K, the increase in potential energy $(1.18 \mathrm{eV})$ is larger than the decrease in kinetic energy $(0.78 \mathrm{eV})$. In Li-Na, however, the decrease in electronic kinetic energy is much larger $(1.2 \mathrm{eV})$, and outweighs the increase in potential energy $(0.7 \mathrm{eV})$. As stated above, the broadening of the bands reinforces the metallic character (electron itineracy) of the amorphous Li-Na structure, inducing the substantial decrease in the average kinetic energy of the electrons. All in all, we conclude that the amorphization in Li-Na nanoalloys has a strong electron shell component, which is not captured by the Gupta calculations (we notice that we do not assume a priori that the differences between Gupta and DFT results have to be ascribed only to the electron shell structure. We rather conclude it a posteriori, from a careful analysis of the DFT results and of the correlation between excess energies and excess radii, which only fails for Li-Na. There are other sources of error in the employed Gupta potentials, such as the omission of a charge transfer contribution to cohesion or the interpolation procedure employed to obtain the heteroatomic parameters. However, the charge transfer is almost negligible in $\mathrm{Li}-\mathrm{Na},{ }^{36}$ and we employed exactly the same interpolation procedure in the four materials. The DFT results point to the electronic shell structure as the only qualitative difference between Li-Na and the other bialkali mixtures).

\section{SUMMARY AND DISCUSSION}

We have reported the putative GM structures of $\mathrm{Na}_{x} \mathrm{Cs}_{55-x}$ and $\mathrm{Li}_{x} \mathrm{Cs}_{55-x}$, as predicted by a first-principles DFT method, and have compared them to the structures of $\mathrm{Li}_{x} \mathrm{Na}_{55-x}$ and $\mathrm{Na}_{x} \mathrm{~K}_{55-x}$ nanoalloys which were reported in a previous paper. ${ }^{36}$ The four bialkali mixtures favor the formation of core-shell segregated structures but span a wide range of size mismatches. By comparing the four systems, we have thus been able to extract some useful trends systematizing the effect of an increasing size mismatch on the structures and stabilities of the alkali nanoalloys. The obtained trends are expected to have some generality for other isovalent metallic nanoalloys with a small degree of charge transfer and a tendency to form core-shell structures.

We summarize first the structural trends. With an increasing size mismatch, we have found that it becomes more and more probable that a single impurity atom modifies the structure of the pure cluster due to the substantial stress generated into the host cluster around a substitutional impurity. For dilute concentrations of the element with smaller size, both A and $\mathrm{B}$ atoms coexist within the cluster core. If the size mismatch is small enough, as in Li-Na, a partially alloyed core is favored. ${ }^{36}$ For large size mismatches, the core itself tends to be segregated. This induces an interesting Janus-like pattern, whereby the growth of the shell follows different atomic packing schemes on the A-rich and B-rich sides of the core. As the concentration of the "small" element increases, core-shell structures become strongly favored and remain stable over a wide range of compositions. The Na-K system has a nearly ideal size mismatch for the formation of core-shell structures with perfect pIh packing. For larger size mismatches, pIh packing becomes frustrated because a perfect pIh epitaxy is no longer possible for steric reasons. In some cases, this may lead to a reconstruction of the structure of the core, which adopts a less dense decahedral packing in order to better adapt to the shell. If the size mismatch is smaller than optimal, as in the Li-Na system, frustration of the pIh packing results in a partial amorphization of the structure, driven by the strong tendency of the surface bonds to contract. This amorphization can be viewed as a synergistic effect as it does not occur in the pure metal clusters. Finally, we have found that electron shell effects are more important the smaller the size mismatch, so that Li-Na nanoalloys adopt prolate shapes (in agreement with jellium model predictions for clusters with 55 electrons) over a wider range of compositions as compared to the rest of systems. For large size mismatches, the preferred cluster shape is 
dictated instead by geometric packing arguments, and it can be either strongly oblate (Na-Cs) or highly spherical (Li-Cs).

Alkali nanoalloys have negative excess energies except for a few compositions. The magic composition $x_{m}$ leading to a minimum in the excess energy has been located for the four systems. $x_{m}$ is found to increase with the size mismatch because of steric crowding effects within the shell. The most reactive mixture is found to be $\mathrm{Li}-\mathrm{Cs}$, followed by $\mathrm{Li}-\mathrm{Na}$, $\mathrm{Na}-\mathrm{Cs}$, and Na-K. The stability ordering of the same four mixtures is exactly the opposite in the bulk limit, where moreover the Li-alkali mixtures are strongly non-reactive. Most of these stability trends can be rationalized with a single parameter, namely, the excess radius, measuring the compactness of the nanoalloy relative to that of an ideal mixture. For each separate system, the magic composition $x_{m}$ coincides with the most compact composition. Also, with the only exception of the Li-Na system, we find that more compact systems are also more stable. We, therefore, propose the use of the excess radius as a very powerful parameter for identifying systematic trends in nanoalloy stability. The good correlation between the stability of the nanoalloy and the strength of the coreshell bonding demonstrates that the interfacial energy (which is a negligible contribution to the total energy of a phaseseparated bulk mixture) is an important factor stabilizing the core-shell segregation pattern at the nanoscale.

The partial amorphization observed in Li-Na nanoalloys induces modifications in the electronic shell structure which result in a substantial stabilization. Both $\mathrm{Li}$ and $\mathrm{Na}$ atoms contribute quite evenly to the HOMO leading to a positive excess VIP. For all other systems, the outermost region of the HOMO is strongly dominated by the shell atoms, and the excess VIP is large and negative. The amorphization also shifts the nearest electron shell closing, from its expected jellium location at 58 electrons to a new value of 56 electrons. $\mathrm{Li}_{19} \mathrm{Na}_{36}$ is, therefore, almost a closed-shell system, which provides an additional electronic stabilization. The ultimate reason for the high stability of the disordered Li-Na structures has been found to be the substantial electronic kinetic energy decrease induced by the amorphization, which is in turn connected with a more continuous density of states and so with an increased metallic character.

The accuracy of the structures predicted by the Gupta empirical potential increases with size mismatch. In fact, for the Li-Cs system, many of the GM structures at the DFT level are within the ten most stable structures predicted by the Gupta potential. This is not surprising, given the bigger relative importance of geometric packing effects for this system. When the size mismatch is smaller and electron shell effects become more important, the predictions of the Gupta potential are less reliable. Nevertheless, the EP model does a very decent job in locating accurate magic compositions for each material and even in predicting the correct relative stabilities of the different materials with the only exception of the Li-Na system. EP models are, therefore, an invaluable tool guiding the much more expensive DFT calculations.

In summary, this work has focused on identifying structural and stability trends in bimetallic core-shell nanoalloys with a fixed size, as a function of composition and size mismatch. Future work will consider the cluster size as an addi- tional variable, in order to identify size-dependent trends and locate the magic sizes of binary metallic nanoalloys.

\section{ACKNOWLEDGMENTS}

This work was supported by the Spanish "Ministerio de Ciencia e Innovación," the European Regional Development Fund, and "Junta de Castilla y León" (Project Nos. FIS200802490/FIS and VA104A11-2). We also acknowledge financial support from the MP0903 COST action.

${ }^{1}$ A. M. Molenbroek and J. K. Norskov, J. Phys. Chem. B 105, 5450 (2001).

${ }^{2}$ H. Yasuda and H. Mori, Phys. Rev. Lett. 69, 3747 (1992).

${ }^{3}$ H. Portales, L. Saviot, E. Duval, M. Gaudry, E. Cottancin, M. Pellarin, J. Lermé, and M. Broyer, Phys. Rev. B 65, 165422 (2002).

${ }^{4}$ J. P. Wilcoxon and P. P. Provencio, J. Am. Chem. Soc. 126, 6402 (2004).

${ }^{5}$ C. Burda, X.-B. Chen, R. Narayanan, and M. A. El-Sayed, Chem. Rev. 105, 1025 (2005)

${ }^{6}$ M. Tchaplyguine, S. Legendre, A. Rosso, I. Bradeanu, G. Öhrwall, S. E. Canton, T. Andersson, N. Märtensson, S. Svensson, and O. Björneholm, Phys. Rev. B 80, 033405 (2009).

${ }^{7}$ R. Ferrando, J. Jellinek, and R. L. Johnston, Chem. Rev. 108, 845 (2008), and references therein.

${ }^{8}$ A. Fortunelli and A. M. Velasco, J. Mol. Struct.: THEOCHEM 487, 251 (1999).

${ }^{9}$ G. Rossi, A. Rapallo, C. Mottet, A. Fortunelli, G. Barcaro, and R. Ferrando, Phys. Rev. Lett. 93, 105503 (2004).

${ }^{10}$ A. Aguado, L. E. González, and J. M. López, J. Phys. Chem. B 108, 11722 (2004); A. Aguado, S. Núñez, and J. M. López, Comput. Mater. Sci. 35, 174 (2006).

${ }^{11}$ A. Aguado and J. M. López, Phys. Rev. B 71, 075415 (2005).

${ }^{12}$ A. Aguado and J. M. López, J. Chem. Theory Comput. 1, 299 (2005).

${ }^{13}$ A. Aguado and J. M. López, Phys. Rev. B 72, 205420 (2005).

${ }^{14}$ J. P. K. Doye and L. Meyer, Phys. Rev. Lett. 95, 063401 (2005).

${ }^{15}$ R. Ferrando, A. Fortunelli, and G. Rossi, Phys. Rev. B 72, 085449 (2005).

${ }^{16}$ G. Barcaro, A. Fortunelli, G. Rossi, F. Nita, and R. Ferrando, J. Phys. Chem. B 110, 23197 (2006).

${ }^{17}$ M. Zhang and R. Fournier, J. Mol. Struct.: THEOCHEM 762, 49 (2006).

${ }^{18}$ R. Ferrando, A. Fortunelli, and R. L. Johnston, Phys. Chem. Chem. Phys. 10, 640 (2008).

${ }^{19}$ E. Hristova, V. G. Grigoryan, and M. Springborg, J. Chem. Phys. 128, 244513 (2008); Eur. Phys. J. D 52, 35 (2009).

${ }^{20}$ F. Calvo, E. Cottancin, and M. Broyer, Phys. Rev. B 77, 121406(R) (2008).

${ }^{21}$ L. O. Paz-Borbón, R. L. Johnston, G. Barcaro, and A. Fortunelli, J. Chem. Phys. 128, 134517 (2008).

${ }^{22}$ F. Chen and R. L. Johnston, ACS Nano 2, 165 (2008).

${ }^{23}$ R. Fournier, J. Comput. Methods Sci. Eng. 8, 331 (2008).

${ }^{24}$ R. Fournier, S. Zamiruddin, and M. Zhang, Can. J. Chem. 89, 1013 (2009).

${ }^{25}$ M. Zhang and R. Fournier, J. Phys. Chem. A 113, 3162 (2009).

${ }^{26}$ D. T. Tran and R. L. Johnston, Phys. Chem. Chem. Phys. 11, 10340 (2009).

${ }^{27}$ L. Delfour, J. Creuze, and B. Legrand, Phys. Rev. Lett. 103, 205701 (2009).

${ }^{28}$ J. A. Reyes-Nava, J. L. Rodríguez-López, and U. Pal, Phys. Rev. B 80, 161412(R) (2009).

${ }^{29}$ I. Parsina and F. Baletto, J. Phys. Chem. C 114, 1504 (2010).

${ }^{30}$ P. S. West, R. L. Johnston, G. Barcaro, and A. Fortunelli, J. Phys. Chem. C 114, 19678 (2010).

${ }^{31}$ F. Delogu, J. Phys. Chem. C 114, 19946 (2010).

${ }^{32}$ D. Bochicchio and R. Ferrando, Nano Lett. 10, 4211 (2010).

${ }^{33}$ G. Zanti and D. Peeters, J. Phys. Chem. A 114, 10345 (2010).

${ }^{34}$ S. Núñez and R. L. Johnston, J. Phys. Chem. C 114, 13255 (2010).

${ }^{35}$ Y. Gao, N. Shao, Y. Pei, and X. C. Zeng, Nano Lett. 10, 1055 (2010).

${ }^{36}$ A. Aguado and J. M. López, J. Chem. Phys. 133, 094302 (2010).

${ }^{37}$ G. Barcaro, A. Fortunelli, M. Polak, and L. Rubinovich, Nano Lett. 11, 1766 (2011).

${ }^{38}$ M. Cerbelaud, R. Ferrando, G. Barcaro, and A. Fortunelli, Phys. Chem. Chem. Phys. 13, 10232 (2011).

${ }^{39}$ S. Xiong, W. Qi, B. Huang, and M. Wang, ChemPhysChem 12, 1317 (2011).

${ }^{40}$ M. Molayem, V. G. Grigoryan, and M. Springborg, J. Phys. Chem. C 115, 7179 (2011)

${ }^{41}$ X. Zhang and A. Zunger, Phys. Rev. Lett. 104, 245501 (2010). 
${ }^{42}$ D. J. Wales and J. P. K. Doye, J. Phys. Chem. A 101, 5111 (1997).

${ }^{43}$ D. J. Wales, Energy Landscapes (Cambridge University Press, Cambridge, England, 2003).

${ }^{44}$ R. P. Gupta, Phys. Rev. B 23, 6265 (1981).

${ }^{45}$ V. Rosato, M. Guillopé, and B. Legrand, Philos. Mag. A 59, 321 (1989).

${ }^{46}$ F. Cleri and V. Rosato, Phys. Rev. B 48, 22 (1993).

${ }^{47}$ Y. Li, E. Blaisten-Barojas, and D. A. Papaconstantopoulos, Phys. Rev. B 57, 15519 (1998).

${ }^{48}$ J. R. Christman and H. B. Huntington, Phys. Rev. 153, 217 (1967).

${ }^{49}$ M. Sicher, S. Mohr, and S. Goedecker, J. Chem. Phys. 134, 044106 (2011).

${ }^{50}$ W. Kohn and L. J. Sham, Phys. Rev. 140, 1133A (1965).

${ }^{51}$ P. Hohenberg and W. Kohn, Phys. Rev. 136, 864B (1964).

${ }^{52}$ J. M. Soler, E. Artacho, J. D. Gale, A. García, J. Junquera, P. Ordejón, and D. Sánchez-Portal, J. Phys.: Condens. Matter 14, 2475 (2002).

${ }^{53}$ J. P. Perdew and A. Zunger, Phys. Rev. B 23, 5048 (1981).

${ }^{54}$ D. M. Ceperley and B. J. Alder, Phys. Rev. Lett. 45, 566 (1980).

${ }^{55}$ R. Hamann, M. Schlüter, and C. Chiang, Phys. Rev. Lett. 43, 1494 (1979).

${ }^{56}$ L. Kleinman and D. M. Bylander, Phys. Rev. Lett. 48, 1425 (1982).

${ }^{57}$ D. L. Hill and J. A. Wheeler, Phys. Rev. 89, 1102 (1953).
${ }^{58}$ C. F. Guerra, J.-W. Handgraaf, E. J. Baerends, and F. M. Bickelhaupt, J. Comput. Chem. 25, 189 (2003).

${ }^{59}$ R. F. W. Bader, Atoms in Molecules. A Quantum Theory (Clarendon, Oxford, 1990)

${ }^{60}$ M. A. Spackman and E. N. Maslen, J. Phys. Chem. 90, 2020 (1986); Aust. J. Phys. 38, 273 (1985).

${ }^{61}$ Y. H. Chui and K. Y. Chan, Phys. Chem. Chem. Phys. 5, 2869 (2003).

${ }^{62}$ S. Kümmel, M. Brack, and P.-G. Reinhard, Phys. Rev. B 62, 7602 (2000).

${ }^{63}$ A. Aguado and O. Kostko, J. Chem. Phys. 134, 164304 (2011).

${ }^{64}$ See supplementary material at http://dx.doi.org/10.1063/1.3645105 for the files containing the atomic coordinates and point group symmetries of the putative GM structures located in this work, at both EP and DFT levels of theory. We also include results for the average bond lengths in alkali nanoalloys.

${ }^{65}$ A. Aguado, J. Phys. Chem. C 115, 13180 (2011).

${ }^{66}$ T. B. Massalski and H. Okamoto, Binary Alloy Phase Diagrams (ASM International, Materials Park, OH, 1986).

${ }^{67}$ J. M. Soler, M. R. Beltrán, K. Michaelian, I. L. Garzón, P. Ordejón, D. Sánchez-Portal, and E. Artacho, Phys. Rev. B 61, 5771 (2000). 